\title{
Vehicle Coordination and Configuration in High-powered Automated Vehicle Storage and Retrieval Systems
}

\author{
Andreas Habl*, Thomas Lienert, Germain Pradines, Johannes Fottner \\ Chair of Materials Handling, Material Flow, Logistics, Technical University of Munich, Boltzmannstraße 15, \\ 85748 Garching bei München, Germany; *andreas.habl@tum.de
}

SNE 30(4), 2020, 139-144, DOI: 10.11128/sne.30.tn.10532 Received: August 1, 2020 (Selected ASIM SPL 2019 Postconf. Publ.); Revised: Oct. 13, 2020; Accepted: Oct. 20, 2020 SNE - Simulation Notes Europe, ARGESIM Publisher Vienna ISSN Print 2305-9974, Online 2306-0271, www.sne-journal.org

Abstract. The growing demand for increased flexibility and throughput capacity of automated warehouses is driving the implementation of rail-guided automated vehicle storage and retrieval systems (AVSRSs). A new type of AVSRS is capable of further increased performance by utilizing multiple vehicles along one rail instead of using only a single vehicle per aisle and tier. In this work, we introduce the shuttle vehicle scheduling problem (SVSP) in AVSRSs and present methods to solve the SVSP. We conduct a series of simulation experiments to show the performance improvement of horizontal transportation in various configurations of this new type of AVSRS and discuss main benefits of deploying high-powered AVSRSs.

\section{Introduction}

Automated vehicle storage and retrieval systems (AVSRSs), also known as shuttle systems, provide a flexible and efficient way for storing small transportation units. Due to the complete decoupling of horizontal and vertical transports executed by shuttle and lift vehicles, AVSRSs with aisle- and tier-captive shuttle vehicles achieve, in combination with unit lifts, the highest throughput capacity. Depending on the warehouse geometry in this version of AVSRS, throughput is limited by either the lift or the shuttle vehicles, i.e., the number of tiers and the length of the aisles [1]. By deploying additional shuttle vehicles on each tier as well as lift vehicles in each shaft, a further increase in throughput can be achieved in such systems if the coordination of several vehicles is successful. This results in a new version of
AVSRS in the field of high performance that can meet the increasing requirements in terms of dynamics and flexibility of AVSRSs. Beyond the increased throughput of newly planned high-powered AVSRSs, existing systems can also be adapted flexibly and with little effort by deploying additional vehicles that can react to changing conditions.

In this work, the potential of high-powered AVSRSs is revealed by means of the horizontal transport on one tier of an aisle. First, an overview of existing research literature in the field of AVSRSs is presented. In order to conduct robust and efficient operations of several shuttle vehicles on a tier, strategies for the coordination of the vehicles are required. After the problem statement, solution approaches are presented and illustrated by means of a selected control strategy. The implementation of the control algorithm in a simulation model allows for subsequent performance testing, in various configurations, of a tier in an aisle.

\section{Literature Review}

The throughput calculation of AVSRSs that is associated with the configuration and design is the subject of numerous scientific studies and can be carried out using either analytical or simulation-based models. For example, the FEM Guideline 9.860 contains an analytical model for calculating the throughput of AVSRSs with vehicle and unit lifts. In particular, special cases such as double-deep storage or the use of several unit lifts in the aisle are considered [2]. Analytical models for AVSRSs with unit lifts and one shuttle vehicle on each tier are developed in [3] and [4]. The modeling of the individual tiers is based on queuing systems and is validated by means of discrete event simulation. The analytical determination of the throughput is, therefore, a suitable method, although it is mainly used for basic systems. 
[5] develops a tool that enables the throughput calculation of various system configurations by using the Monte Carlo simulation.

[6] presents a simulation model for analyzing, in a short time, rack configurations with several lifts per aisle, in order to identify the optimal configuration. [7] conducts a simulation study to investigate the effects of different variables on the achievable throughput capacity of AVSRSs with unit lifts. Hence, a simulation-based throughput calculation is increasingly used if a large number of configurations and more complex systems are being investigated.

In principle, the throughput of AVSRSs is significantly influenced by their control. However, especially in the case of basic configurations of AVSRSs with unit lifts, the transport processes often do not require complex controls, or can often be defined by simple priority rules. An exception is the AVSRS with unit lifts considered in [8] with two lift vehicles moving in the same lift shaft. The vehicles are controlled by a block sequencing algorithm that optimizes the sequence of the transport jobs with the help of heuristics. [9] takes up this approach and supplements it by taking into account the acceleration and deceleration processes of the vehicles. In lift configurations with more than one lift vehicle per shaft, simple priority rules reach their limits and more complex control strategies are required to enable an efficient and robust operation.

Until now, AVSRSs with several vehicles on a tier have not been considered in literature, whether in regard to the achievable throughput or to the necessary control strategies.

\section{Coordination of Multiple Shuttle Vehicles on a Tier}

The coordination of several shuttle vehicles on a tier is a challenge for the control applied. In the following, the associated problems and possible solutions are presented and, finally, a selected control algorithm is described.

\subsection{Problem Statement}

In high-powered AVSRSs, several shuttle vehicles that move along the same railing system are available for horizontal transport on the individual tiers of an aisle. To ensure robust transport processes, it is necessary to coordinate the shuttle vehicles in such a way that block events and collisions are prevented.
At the same time, the control must ensure that waiting times and empty driving times are minimized, thus making the transport processes efficient. This challenge, which we define as Shuttle Vehicle Scheduling Problem (SVSP), is divided into the three successive problems of job allocation, sequencing and execution.

While the presented SVSP has not yet been considered, the Crane Scheduling Problem (CSP) is a comparable scheduling problem that occurs in the area of gantry and overhead crane control and has often been discussed in literature.

In addition to the CSP, the SVSP can also be regarded as a special case of general machine scheduling - it can, thus, be assigned to NP-hard problems and formulated equivalently [10]:

$n$ jobs $\left\{j_{1}, j_{2}, \ldots, j_{n}\right\}$ must be processed, whereby

$m$ shuttle vehicles $\left\{s_{1}, s_{2}, \ldots, s_{m}\right\}$ are available.

Basically, the optimization aims at minimizing the processing time of the considered jobs and, thus, maximizing the throughput on the tier. The following questions must, therefore, be answered in the SVSP:

- Which shuttle vehicles are, in principle, allowed to carry out the job concerned? (preselection of the shuttle vehicles)

- Which shuttle vehicle takes over which job and in which sequence or at what time? (allocation of the jobs to the shuttle vehicles)

\subsection{Solution Approaches for the Shuttle Vehicle Scheduling Problem}

In order to solve the SVSP on the tier, we rely on work carried out in the area of CSP. [11] evaluates and classifies possible strategies for preselection and allocation (Figure 1). Adapted to the SVSP on the tier, the following preselection strategies can be formulated:

- Location-based restriction: The tier is divided into zones that are assigned to the individual shuttle vehicles. Jobs that span several zones can, thus, only be fully processed by several vehicles.

- Task-related restriction: Shuttle vehicles are either responsible for the supply and disposal of one or more lifts or carry out assisting transport tasks, such as relocation or preliminary transports.

- No restriction: All jobs can be processed by all shuttle vehicles. 
The preselection assigns each job $j_{i}$ of the job list $J$ to all shuttle vehicles which are in principle able to execute it, i.e., each shuttle vehicle $s_{i}$ receives a list of jobs $j_{i} \ldots j_{j} \in J$. The individual lists can overlap depending on the preselection strategy. This is the basis for the second step, in which the jobs are finally assigned to the shuttle vehicles using an allocation method, and the sequence for the job execution is determined for each vehicle.

According to [11], one option to conduct the job allocation is by means of block sequencing or dynamic sequencing. In this case, the concepts of block sequencing and dynamic sequencing, in conjunction with rolling scheduling, are both based either on optimal solution methods or on approximate solutions using heuristics. In both cases, a defined number of jobs are planned in parallel (simultaneous assignment). The use of optimization methods, such as the Branch-and-Bound method, guarantees that the shortest possible schedule is found for the different jobs. Heuristics and Metaheuristics aim to obtain a solution as close as possible to the optimum in acceptable computing time [10].

Another approach for the job allocation is the use of priority rules in dynamic sequencing. In this case, only the next pending job is assigned to a shuttle vehicle (successive assignment) that

- has been the least used until now,

- completes its current job the next and becomes available,

- is closest to the job start location, or

- is chosen randomly.

\subsection{Control Algorithm for Coordinating Multiple Shuttle Vehicles}

The investigation of a potential performance increase on the tier of an aisle in AVSRSs firstly requires the selection and implementation of a control algorithm. For this purpose, no restriction, i.e., each shuttle vehicle is able to carry out any job on the tier (see section 2.2), is chosen for preselection.

For job allocation, the block sequencing algorithm based on [12], originally developed for the control of overhead cranes, is used and is now adapted to the application on the tier of a high-powered AVSRS.

Functionality. Based on block sequencing and, thus, depending on the selected block size, a number of jobs are planned in parallel (job allocation) and then executed by the shuttle vehicles.
After the last job is completed, the job block is removed from the job list and the next block is planned in the next iteration.
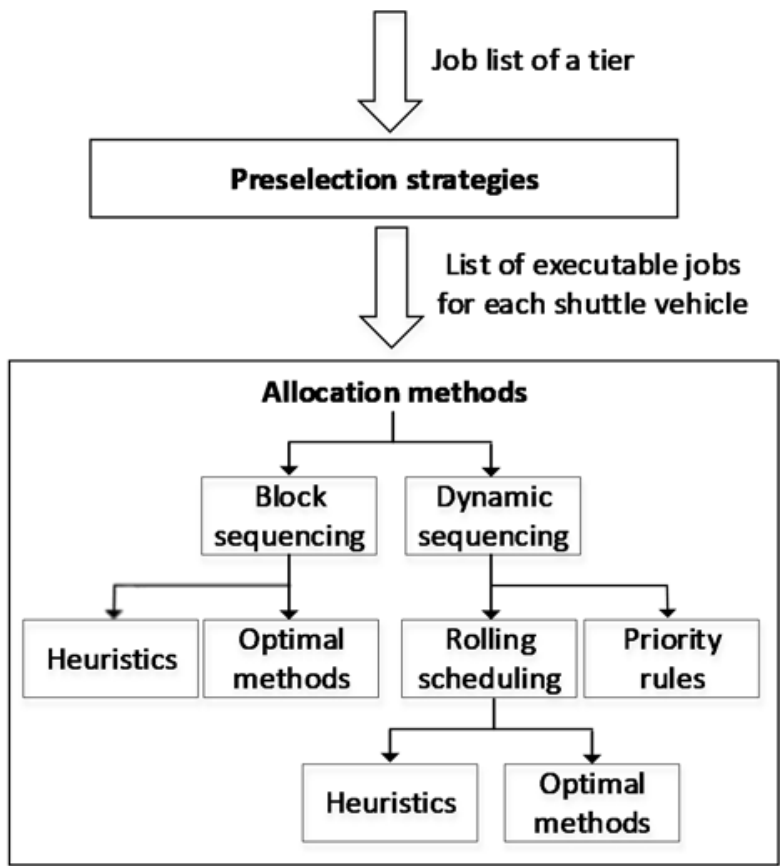

Figure 1: Classification of strategies to solve the SVSP-based on [11].

Job allocation. The job allocation of a block consists in assigning, with subsequent sequencing, a job to a shuttle vehicle by determining a start time for this job. Then, the algorithm iteratively spans a solution tree, whose nodes contain all possible partial schedules. In each iteration, the algorithm tries to add further pairs (shuttle vehicle - job) to the existing nodes and to append the newly created nodes, until the respective schedule is complete. The complete schedules correspond to the leaves of the solution tree and represent the feasible combinations of pairs. Finally, the schedule that is complete and requires the smallest processing time is selected for job execution.

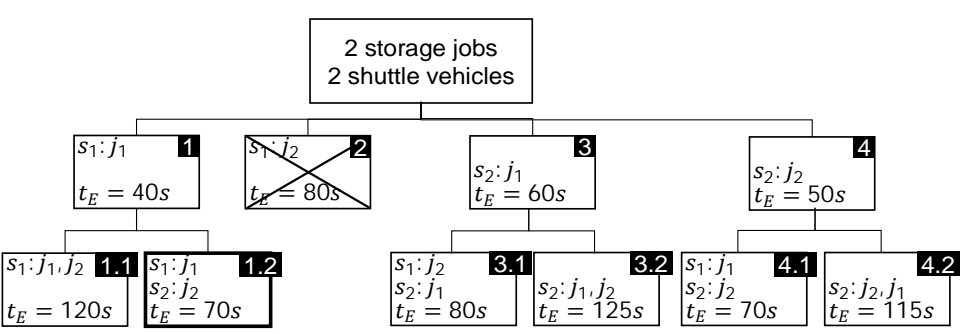

Figure 2: Examplary solution tree with two jobs and two shuttle vehicles. 
Figure 2 shows a basic example of the assignment of two jobs to two shuttle vehicles. In the first iteration, there are four possibilities to assign a job to a shuttle vehicle. For the next iteration, the node with the smallest processing time is selected (schedule 1) and completed by two assignment alternatives.

Then, an analogous procedure is carried out for nodes 3 and 4 , whereas node 2 is no longer pursued as it is dominated by schedule 1.2. Finally, the schedule with the smallest processing time is selected (schedule 1.2) and transferred to execution.

Job execution. After the scheduling of the block, the execution is carried out by the shuttle vehicles. For each job, it is first determined whether it can be executed immediately or whether waiting times must be observed in order to prevent block events with other shuttle vehicles. If the job has to be executed immediately, the trajectory planning and travel to the starting point of the job take place. Otherwise, one or more waiting positions or intermediate stops must first be determined and driven to in order to avoid interferences with other shuttle vehicles.

Applied heuristics. The number of possible solutions or schedules grows exponentially with the block size and causes the computing effort to increase accordingly. To limit the computing time, a heuristic is used that

- deletes incomplete schedules whose processing times are already bigger than that of a complete schedule (see Figure 2),

- removes a defined number of nodes from the tree, which has a certain total amount of nodes. Nodes or schedules with the smallest number of scheduled jobs or - if the number of jobs is identical - requiring a higher processing time are removed.

\section{Simulation-based Analysis}

A simulation model was created using the discrete event simulation environment Plant Simulation to investigate the throughput of a tier in a high-powered AVSRS. The model allows different configurations of the tier and aisle respectively, and is afterwards described. The results are subsequently presented and evaluated.

\subsection{Simulation Model and Parameters}

The simulation model contains one tier of an aisle in an AVSRS and has the following components (Figure 3):
- Rack with storage bins (free or occupied by a transportation unit)

- Railing system with an extension on either side of the tier for vehicle evasion (evasion buffer)

- Shuttle vehicles (with kinematic parameters based on [2])

- Lifting systems with two transfer buffers for storage and retrieval

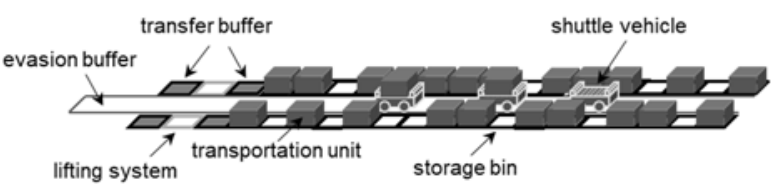

Figure 3: Section of a tier in an aisle of a high-powered AVSRS.

The analyzed configurations of the tier in the simulation study are shown in Table 1, where the lifting systems serve as the boundary of the simulation model. The listed configurations contain 200 storage bins (100 storage bins in each longitudinal direction of the aisle), as this is a common number of bins per tier in practice, and differ in the number of deployed lifting systems and their positioning within the aisle.

\begin{tabular}{llll}
\hline $\begin{array}{l}\text { Configura- } \\
\text { tion }\end{array}$ & $\begin{array}{l}\text { Number of } \\
\text { lifting sys- } \\
\text { tems }\end{array}$ & $\begin{array}{l}\text { Position of } \\
\text { lifting sys- } \\
\text { tems }\end{array}$ & $\begin{array}{l}\text { Number of } \\
\text { storage bins }\end{array}$ \\
\hline 1 & 2 & $\begin{array}{l}\text { Start of } \\
\text { aisle }\end{array}$ & 200 \\
\hline 2 & 2 & $\begin{array}{l}\text { Center of } \\
\text { aisle }\end{array}$ & 200 \\
\hline 3 & 4 & $\begin{array}{l}\text { Start and } \\
\text { center of } \\
\text { aisle }\end{array}$ & 200 \\
\hline 4 & 4 & $\begin{array}{l}\text { Start and } \\
\text { end of aisle }\end{array}$ \\
\hline
\end{tabular}

Table 1: Considered configurations of the tier.

For the simulation-based throughput analysis, the number of shuttle vehicles goes from 1 to 5 in each configuration. The resulting 20 experiments are performed with five replications.

\subsection{Results}

In this section, the results of the conducted simulation study are presented and discussed on the basis of the described simulation model. 
Comparison of different configurations. Figure 4 shows the obtained throughput with respect to the number of deployed shuttle vehicles for each configuration. Basically, throughput increases initially by deploying additional shuttle vehicles and decreases when four or five shuttle vehicles are deployed on the tier. This relies on the rising evasive moves, especially at the lifting systems, when the number of vehicles is increasing. Although unneeded vehicles could park in the evasion buffers, meaning that throughput should not be reduced by the further increase in the number of vehicles, this possibility is not taken into account by the heuristics in the scheduling process.

By positioning the two lifting systems in the center of the aisle (configuration 2), the shuttle vehicles can approach the lifting systems from both sides without timeconsuming evasive moves, thus enabling the highest throughput throughout. When placing the two lifting systems at the beginning of the aisle (configuration 1), significantly longer distances and interferences between shuttle vehicles at the lifting systems lead to the lowest throughput. A slight increase in throughput is achieved when two lifting systems are positioned at the end of the aisle, as well as two lifting systems at the beginning of the aisle (configuration 4). A further increase in throughput can be achieved by positioning two further lifting systems in the center of the aisle (configuration 3), since shuttle vehicles can now approach the lifting systems from both sides again.

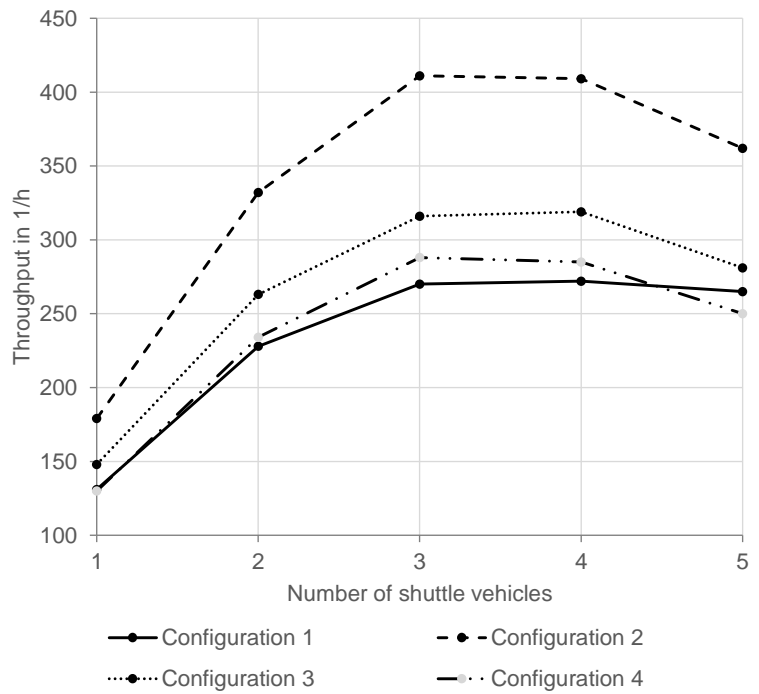

Figure 4: Achieved throughput depending on the number of shuttle vehicles and aisle configurations.

\section{Increased performance by deploying additional} shuttle vehicles. Figure 5 shows the increase in performance for the number of shuttle vehicles concerned by applying configuration 2. Consequently, the deployment of one additional shuttle vehicle increases the throughput produced by the deployment of a single shuttle vehicle by $85 \%$. Two additional shuttle vehicles further increase the throughput by about $24 \%$, until finally, by deploying five vehicles, there is a reduction in throughput of about $-11 \%$.

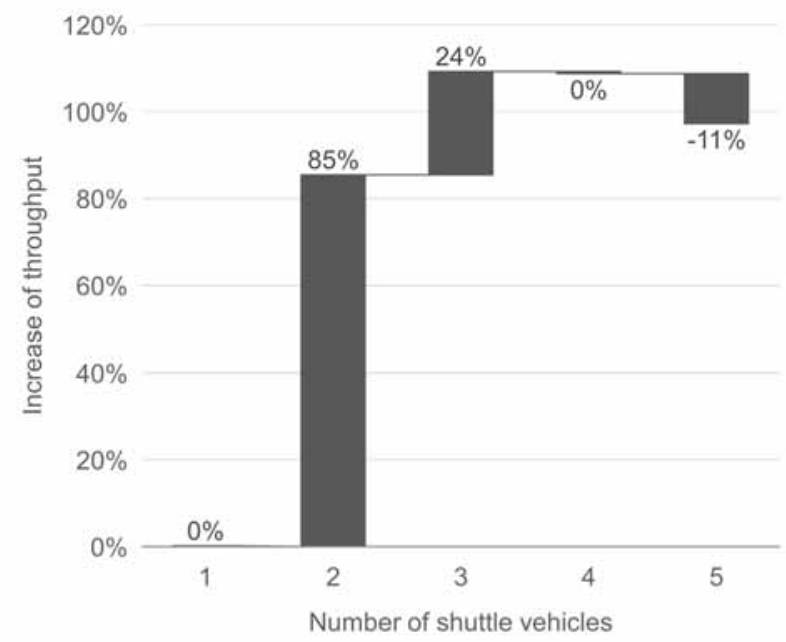

Figure 5: Increased capacity by deploying further vehicles on a tier of an aisle (configuration 2).

\section{Conclusion and Outlook}

High-powered AVSRSs represent a new version of AVSRS with increased flexibility and dynamics. However, these systems require a much more complex control to run the system in a robust and efficient manner. In this sense, the SVSP was introduced for this work; solution approaches, based on existing literature in the scheduling of gantry and overhead cranes, were also presented. By implementing the control algorithm in a simulation model, an increase in throughput was demonstrated by deploying several shuttle vehicles on a tier. Depending on the configuration of the aisle, the throughput decreases again when deploying too many shuttle vehicles. Even if the applied heuristic limits a complete enumeration and the exponential increase of the solution space, the control algorithm requires long computing times due to block sequencing, especially with an increase in the block size and and in the number of shuttle vehicles. 
Further control algorithms could provide a remedy through alternative solution methods and, at the same time, enable real-time capability of scheduling in order to apply the method found in industrial practice. As demonstrated in the simulation study, the number and positioning of the lifting systems within the aisle have a decisive influence on the degree of increase in throughput. Equivalent to horizontal transport, the performance of vertical transport could also be improved by deploying several lift vehicles in a common shaft. Since the SVSP also occurs in a modified form, the same control algorithms can be applied. However, the interfaces between horizontal and vertical transport play a key role, since they have to be coordinated.

\section{References}

[1] Eder M, Kartnig G. Throughput analysis of S/R shuttle systems and ideal geometry for high performance. FME Transaction. 2016; 44: 174-179. doi: $10.5937 / \mathrm{fmet} 1602174 \mathrm{E}$

[2] FEM European materials handling federation. Guideline Cycle time calculation for automated vehicle storage and retrieval systems (9860); 2017.

[3] Marchet G, Melacini M, Perotti S et al. Analytical model to estimate performances of autonomous vehicle storage and retrieval systems for product totes. International Journal of Production Research. 2012; 50: 7134-7148. doi: 10.1080/00207543.2011.639815

[4] Eder M, Kartnig G. Durchsatzoptimierung von ShuttleSystemen mithilfe eines analytischen Berechnungsmodells. 12. Fachkolloquium der Wissenschaftlichen Gesellschaft für Technische Logistik e.V.; 2014 Sep; Stuttgart. 171-177.
[5] Trummer W, Jodin D. Welche Leistung haben Shuttles? Hebezeuge Fördermittel. 2014; 54: 240-242.

[6] Zhao N, Luo L, Zhang S et al. An efficient simulation model for rack design in multi-elevator shuttle-based storage and retrieval system. Simulation Modelling Practice and Theory. 2016; 67: 100-116.

[7] Lerher T, Ekren YB, Sari Z. Simulation analysis of shuttle based storage and retrieval systems. International Journal of Simulation Modelling. 2015; 14: 48-59. doi: 10.2507/IJSIMM14(1)5.281

[8] Carlo HJ, Vis IFA. Sequencing dynamic storage systems with multiple lifts and shuttles. International Journal of Production Economics. 2012; 140: 844-853.

[9] Zhao N, Luo L, Lodewijks G. Scheduling two lifts on a common rail considering acceleration and deceleration in a shuttle based storage and retrieval system. Computers \& Industrial Engineering. 2018; 124: 48-57. doi: 10.1016/j.cie.2018.07.007

[10] MacCarthy BL, Liu J. Addressing the gap in scheduling research: A review of optimization and heuristic methods in production scheduling. International Journal of Production Research. 1993; 31: 59-79. doi: 10.1080/00207549308956713

[11] Kemme N. RMG Crane scheduling and stacking. In: Böse JW, editor. Handbook of Terminal Planning, vol 49. Springer New York, New York, NY; 2011. p 27131.

[12] Peterson B, Harjunkoski I, Hoda S et al. Scheduling multiple factory cranes on a common track. Computers \& Operations Research. 2014; 48: 102-112. doi: 10.1016/j.cor.2014.03.005 